\title{
Topical pharyngeal anesthesia for unsedated esophagogastroduodenoscopy procedure in sick patients
}

\begin{abstract}
The aim of this study was to evaluate and compare the clinical efficacy of topical pharyngeal anesthesia for unsedated esophagogastroduodenoscopy (UEGD) procedure in sick (American Society of Anesthesiologists [ASA] physical status III-IV) and nonsick (ASA physical status I-II) patients in a teaching hospital in Thailand. We undertook a retrospective review of the anesthesia or sedation service records of adult patients who underwent UEGD procedures from October 2008 to September 2009. All patients were classified into two groups according to the ASA physical status. In group A, the patients had ASA physical status I-II, while in group B, the patients had ASA physical status III-IV. The primary outcome variable of the study was the successful completion of the procedure. The secondary outcome variables were anesthesia and procedure-related adverse events during and immediately after the procedure. After matching age, gender, weight, height and duration of procedure, there were 566 adult patients who underwent UEGD procedures by using topical pharyngeal anesthesia. Of these, 287 patients were in group A and 279 patients were in group B. There were no significant differences in age, gender, weight, height and duration of UEGD procedure between the two groups. All patients in both groups successfully completed the procedure except four patients in group A, and one patient in group $\mathrm{B}(\mathrm{P}=0.188)$. Anesthesia and procedure-related adverse events in both groups were not significantly different. All adverse events were transient and mild degree with no adverse sequelae. Topical pharyngeal anesthesia for UEGD procedure in sick patients by trained anesthetic personnel with appropriate monitoring was safe and effective. The clinical efficacy of this technique in sick patients was not different or worse than in nonsick patients. Serious adverse events were rare in our population.
\end{abstract}

Volume 4 Issue 2 - 2016

Somchai Amornyotin, Wiyada Chalayonnawin, Siriporn Kongphlay

Department of Anesthesiology and Siriraj GI Endoscopy Center, Faculty of Medicine Siriraj Hospital, Mahidol University, Thailand

Correspondence: Somchai Amornyotin, Department of Anesthesiology and Siriraj GI Endoscopy Center, Faculty of Medicine Siriraj Hospital, Mahidol University, 2, Siriraj, Bangkoknoi, Bangkok 10700, Thailand, Tel +662419 7990, Fax +662 4I I 3256, Email somchai.amo@mahidol.ac.th

Received: November 19,2015 | Published: January 29, 2016

Keywords: topical, pharyngeal anesthesia, esophagogastroduodenoscopy, unsedated, efficacy, safety, sick, american society of anesthesiologists (ASA)

\section{Abbreviations:}

UEGD, esophagogastroduodenoscopy; ASA, American society of anesthesiologists; SD, standard deviation; \%, percentage

\section{Introduction}

Unsedated esophagogastroduodenoscopy (UEGD) is generally safe and can be well tolerated. ${ }^{1,2}$ The proportion of patients undergoing diagnostic EGD without sedation has been increasing over the past decade, and previous studies suggest that many patients who receive adequate information about the procedure now choose not to have sedation. ${ }^{3,4}$ In Thailand, most of diagnostic and screening EGDs are performed without sedation. Topical pharyngeal anesthesia is often used as premedication for EGD procedure. ${ }^{5,6}$ In a recent metaanalysis, topical pharyngeal anesthesia before EGD with sedation is shown to improve ease of endoscopy and patient tolerance. Pharyngeal anesthesia is also often used in UEGD and is thought to improve patient tolerance. ${ }^{7}$ There have not been any studies directly comparing the efficacy and safety of topical lidocaine for pharyngeal anesthesia when the topical lidocaine is used as a single agent for UEGD procedure between sick (ASA physical status III-IV) and nonsick (ASA physical status I-II) patients.

\section{Materials and methods}

\section{Patients}

The adult patients who underwent UEGD procedure at Siriraj GI Endoscopy Center, Faculty of Medicine, Siriraj Hospital between
October 2008 to September 2009 were enrolled in the present study. Inclusion criteria were the adult patients (age $\geq 20$ years) who underwent ERCP procedure by using topical pharyngeal anesthesia technique. UEGD procedures performed in operating rooms and the procedures performed with sedation, or procedures performed under general anesthesia were excluded.

\section{Study design}

This study was a retrospective descriptive study. All elderly patients were classified into two groups according to their ASA physical status. In group A, the patients had ASA physical status I-II. In group B, the patients had ASA physical status III-IV. The primary outcome variable of the study was the successful completion of the procedure. A failed procedure was defined as a procedure that could not be completed by using the PBDS technique when under deep sedation or when sedation-related serious adverse events, such as severe hypoxemia (oxygen saturation $\left[\mathrm{SpO}_{2}\right]<85 \%$ for longer than 3 minutes, which cannot be relieved by airway management) or severe cardiorespiratory instability occur. The secondary outcome variables were sedation and procedure-related adverse events during and immediately after the procedure.

\section{Endoscopy procedure}

The procedure was performed by either gastroenterology fellow supervised by staff attending physician or by the staff endoscopist. Olympus video esophagogastroduodenoscopy (GIF-Q 180, Olympus Corporation, Tokyo, Japan) was used for all EGD procedures. The 
success rate in both groups was recorded. The successful completion of the procedure was defined as completion of the procedure as intended without additional sedation or general anesthesia once the procedure had started.

\section{Topical pharyngeal anesthesia}

All pharyngeal anesthesia was administered by the nurse anesthetist or anesthesiology resident supervised by the staff anesthesiologist in the pre-procedural room. Viscous lidocaine solution (Xylocaine ${ }^{\circledR} 2 \%$ viscous, Astra Zeneca) and/or lidocaine spray (Xylocaine ${ }^{\circledR} 10 \%$ spray, Astra Zeneca) was utilized for pharyngeal anesthesia. Each patient was monitored in standard manner for blood pressure, heart rate, heart rhythm with single lead electrocardiogram, and oxygen saturation with pulse oximetry. No other premedications were administered before the procedure. When, the procedure was a failure, intravenous sedation was carried out. Sedative/analgesic agents used in both groups were propofol, fentanyl, and/or midazolam.

\section{Anesthesia-related adverse events}

All anesthesia-related adverse events were recorded. Anesthesiarelated adverse events were defined as follows:

a. Hypertension or Hypotension (increase or decrease in blood pressure by $25 \%$ from baseline).

b. Tachycardia or Bradycardia (increase or decrease in heart rate by $25 \%$ from baseline).

c. Any cardiac arrhythmias; hypoxia (oxygen desaturation, $\mathrm{SpO}_{2}<90 \%$ ); airway obstruction.

\section{Statistical analysis}

Results were expressed as mean \pm standard deviation (SD) or percentage (\%), when appropriate. Comparisons between group A and $\mathrm{B}$ were compared by using $\chi 2$ tests (for categorical variables), $\chi^{2}$ square tests for trend (for ordinal variables), and two-sample independent t-test (for continuous variables). The statistical software package SPSS for Windows (v11; SPSS, Inc, Chicago, IL) was used to analyze the data. All statistical comparisons were made at the twosided $5 \%$ level of significance.

\section{Results}

After matching age, gender, weight, height and duration of procedure, there were 566 adult patients who underwent UEGD procedure by using topical pharyngeal anesthesia. Of these, 287 patients had ASA physical status I-II (Group A) and 279 patients had ASA physical status III-IV (Group B). Table 1 summarizes the characteristics of patients, duration of procedure and endoscopy success rate of the two groups. There were no statistically significant differences in age, gender, weight, height and duration of procedure between the two groups. Although, the mean ages in years in group A $(49.6 \pm 16.5$ years) was slightly higher than in group B $(56.2 \pm 13.4$ years), however, there was not significantly different $(p=0.120)$. All UEGD procedures were successfully completed except four patients in group $\mathrm{A}$, and one patient in group $\mathrm{B}(\mathrm{P}=0.188)$. All failed procedures were successfully completed by using intravenous sedation with the combination of propofol, fentanyl and midazolam.

Table 2 shows the anesthesia and procedure-related adverse events during and immediately after endoscopy in the sick and nonsick groups. Overall, 91 patients $(31.6 \%)$ in group A and 71 patients $(25.6 \%)$ in group B experienced adverse events. All adverse events in the nonsick patients were relatively greater than in the sick patients. However, there were no statistically significant differences in overall, anesthesia-related and procedure-related adverse events between the two groups. Additionally, there were no procedure-related complications in either group.

Table I Characteristics of patients, duration of procedure and endoscopy success

\begin{tabular}{llll}
\hline & $\begin{array}{l}\text { Group A } \\
(\mathrm{n}=287)\end{array}$ & $\begin{array}{l}\text { Group B } \\
(\mathrm{n}=279)\end{array}$ & PValue \\
$\begin{array}{l}\text { Age (yr) (mean, SD) } \\
\text { Gender (\%) }\end{array}$ & $49.6(16.5)$ & $56.2(13.4)$ & 0.120 \\
Male & $178(62.0)$ & $185(66.3)$ & 0.288 \\
Female & $109(38.0)$ & $94(33.7)$ & \\
Weight (kg) (mean, SD) & $56.8(10.9)$ & $59.3(11.8)$ & 0.181 \\
Height (cm) (mean, SD) & $160.4(7.5)$ & $160.5(7.3)$ & 0.262 \\
ASA physical status (\%) & $287(100.0)$ & 0 & \\
I-II & 0 & $279(100.0)$ & \\
III-IV & $11.6(6.6)$ & $11.6(4.7)$ & 0.067 \\
$\begin{array}{l}\text { Duration of procedure (min) } \\
\text { (mean, SD) }\end{array}$ & $283(98.6)$ & $278(99.6)$ & 0.188 \\
Endoscopy success (\%) &
\end{tabular}

Table 2 Anesthesia and procedure-related adverse events during and immediately after endoscopy (n, \%)

\begin{tabular}{llll}
\hline & Group A & Group B & P Value \\
$(\mathrm{n}=279)$ & $71(25.6)$ & 0.100 \\
Overall & $91(31.6)$ & $71(25.6)$ & 0.100 \\
Anesthesia-related & $91(31.6)$ & $28(10.1)$ & 0.059 \\
Tachycardia & $44(15.3)$ & $28(10.1)$ & 0.978 \\
Hypertension & $29(10.1)$ & $14(5.0)$ & 0.518 \\
Tachycardia and hypertension & $18(6.2)$ & $1(0.4)$ & 0.310 \\
Bradycardia & 0 & 0 & 1.000 \\
Procedure-related & 0 & & \\
\hline
\end{tabular}

Group A:ASA physical status I-II; Group B:ASA physical status III-IV. 
Table 3 demonstrates the hemodynamic parameters including systolic and diastolic blood pressure, heart rate and oxygen saturation in both groups. Mean oxygen saturation at the insertion of endoscope in group $\mathrm{B}$ was significantly lower than in group $\mathrm{A}(\mathrm{P}=0.035)$. However, mean oxygen saturation at this time point in both groups was more than $98 \%$. Furthermore, mean heart rate at 10 min after the insertion of endoscope in group A was relatively greater than group B $(\mathrm{P}=0.045)$. Systolic and diastolic blood pressure at all time points in both groups was not significantly different.

Table 3 Hemodynamic parameters: systolic and diastolic blood pressure $(\mathrm{mmHg})$, heart rate (beat/minute) and oxygen saturation $\left(\mathrm{SpO}_{2}, \%\right)\left(\mathrm{Mean}_{\mathrm{S}} \mathrm{SD}\right)$

\begin{tabular}{|c|c|c|c|}
\hline & Group A $(n=287)$ & Group B $(n=279)$ & P Value \\
\hline \multicolumn{4}{|l|}{ Baseline } \\
\hline SBP, DBP & $129.8(\mid 6.8), 75.9(|1| .6)$ & I29.5 (I8.8), $73.7(\mid 2.5)$ & $0.816,0.622$ \\
\hline $\mathrm{HR}, \mathrm{SpO}_{2}$ & 81.0 (I5.5), 98.9 (I.4) & 77.5 (I5.8), $98.6(1.6)$ & $0.123,0.486$ \\
\hline \multicolumn{4}{|c|}{ At insertion } \\
\hline SBP, DBP & I38.3 (20.6), 80.3 (16.2) & I38.6 (24.3), 78.5 (16.5) & $0.208,0.118$ \\
\hline $\mathrm{HR}, \mathrm{SpO}_{2}$ & 90.6 (I8.3), 98.9 (I.4) & 86.1 (19.0), 98.7 (I.7) & $0.205,0.035^{*}$ \\
\hline \multicolumn{4}{|c|}{$5 \mathrm{~min}$ after insertion } \\
\hline SBP, DBP & |38.8 (21.7), 79.1 (16.4) & I38.5 (23.5), $76.0(15.4)$ & $0.631,0.564$ \\
\hline \multicolumn{3}{|c|}{$10 \mathrm{~min}$ after insertion } & $0.063,0.061$ \\
\hline SBP, DBP & |35.| (19.9), $77.6(15.8)$ & | 33.7 (23.7), $75.2(15.0)$ & $0.123,0.367$ \\
\hline $\mathrm{HR}, \mathrm{SpO}_{2}$ & $89.4(16.9), 99.1(1.3)$ & 84.7 (I7.8), 98.9 (I.3) & $0.045^{*}, 0.277$ \\
\hline \multicolumn{4}{|c|}{15 min after insertion } \\
\hline SBP, DBP & I32.I (I8.0), 75.1 (16.8) & I4I.5 (25.0), 76.0 (16.2) & $0.449,0.202$ \\
\hline $\mathrm{HR}, \mathrm{SpO}_{2}$ & 86.8 (I6.8), 98.9 (I.4) & 87.5 (19.0), 98.9 (I.4) & $0.456,0.299$ \\
\hline \multicolumn{4}{|c|}{$20 \mathrm{~min}$ after insertion } \\
\hline SBP, DBP & I26.4 (I8.5), 72.1 (I5.0) & I4I.2 (20.6), 77.4 (15.2) & $0.564,0.390$ \\
\hline $\mathrm{HR}, \mathrm{SpO}_{2}$ & 84.0 (I8.I), 98.7 (I.5) & $81.7(15.3), 98.6(1.5)$ & $0.489,0.620$ \\
\hline
\end{tabular}

GroupA:ASA physical status I-II;Group B:ASA physical status III-IV;SBP:Systolic blood pressure;DBP:Diastolic blood pressure; HR:Heart rate;SPO :Oxygen saturation *Considered to be of statistically significant.

\section{Discussion}

UEGD is considered to be safe, feasible, and well tolerated procedure. In addition, the unsedated procedure is well accepted as demonstrated by patients' willingness to repeat the procedure under similar conditions. ${ }^{1-4,7}$ In healthy patients, there are a number of advantages associated with completing EGD without sedation. These include a decreased incidence of hypoxemia and cardiorespiratory adverse events, a shorter examination time, decreased hospital costs, the ability to work and drive immediately after the procedure. ${ }^{8}$ Generally, the majority of patients would achieve a satisfactory score of home-readiness on or before 1 hour after endoscopic procedure even in the sedated patients. The time to home-readiness by objective evaluation correlated with the type of procedure. ${ }^{9}$ However, limited studies of the efficacy and safety of UEGD in sick patients have been occurred.

Topical pharyngeal anesthesia then becomes important in facilitating patient's tolerance to unsedated EGD. The importance and efficacy of pharyngeal anesthesia has been reviewed in a metaanalysis study favoring topical anesthesia in patients undergoing sedated EGD. ${ }^{7}$ However, the potential benefit from the use of topical anesthetic preparations before EGD remains controversial., ${ }^{3,10,11}$ Two randomized studies concluded that topical lidocaine spray did not facilitate EGD..$^{12,13}$ However, in a randomized double-blind placebo controlled study by Soma Y et al. ${ }^{14}$ also demonstrated that topical pharyngeal anesthesia significantly reduced the risk of discomfort in EGD by $44 \%$ in all patients. Consequently, our previous study demonstrated that UEGD procedure was successfully completed in $93.3 \%$ from the viscous lidocaine and $99.7 \%$ from the lidocaine spray. The use of topical lidocaine as a single agent for pharyngeal anesthesia was safe with rare serious adverse events related to the use of topical lidocaine. ${ }^{2}$

The present study is the first study comparing the efficacy and safety of topical pharyngeal anesthesia for UEGD procedure in the patients with ASA physical status I-II and the patients with ASA physical status III-IV. The primary objective of the study was to evaluate the rate of completion of endoscopy in the sick and nonsick groups. Our result showed that both groups have good overall successful completion rate. Our overall success rate in performing UEGD procedure is better than rates that had been reported. In a study involving US Air Force community, the success rate in performing unsedated EGD was $88 \% .{ }^{15}$ A study from England reported the success rate of 92\%. Failure to tolerate endoscopy was associated with younger age and examination with a standard endoscope. High levels of patient discomfort during the procedure were related with high levels of pre-endoscopic anxiety, younger age and examination with a standard endoscope. ${ }^{16}$ However, the rate of completion of UEGD procedure in the present study is comparable to our previous report. ${ }^{2}$

The factors associated with successful completion of UEGD have been reported. These included older age, lower level of pre-endoscopic apprehension, smaller endoscope diameter, male gender, and having undergone prior unsedated endoscopy. ${ }^{11,16}$ The higher success rate of completed procedure in this study may be due to two factors. First, the majority of the indications of procedure were diagnostic and less invasive therapeutic procedures. Mean duration of these procedures in both groups was 11 minutes. Second, the willingness of unsedated technique for this endoscopic procedure in both groups is relatively high. Although, UEGD is effective in selected patients, however, it provides reduced the endoscopist's satisfaction. Several previous studies showed sedation to accomplish better patient cooperation and satisfaction and a willingness to have it repeated. ${ }^{17,18}$ Unfortunately, this study did not evaluate the tolerance toward unsedated endoscopy, procedural pain as well as the patient and endoscopist satisfaction.

The data regarding the safety of UEGD are limited, and there are no large prospective studies that address safety. Limited studies have reported no serious adverse events in 60 patients $^{19}$ and in 
170 patients ${ }^{20}$ who had unsedated endoscopic procedures. Overall adverse event rate during and immediately after endoscopy in this study was relatively high $(31.6 \%$ in group A and $25.6 \%$ in group B). The majority of these adverse events were anesthesia-related adverse events including tachycardia and hypertension. However, the procedure-related adverse events in both groups were none. A high number of adverse event rates might be due to the definitions of adverse events: hypertension (increase in blood pressure by $25 \%$ from baseline); hypotension (decrease in blood pressure by $25 \%$ from baseline); tachycardia (increase in heart rate by $25 \%$ from baseline); bradycardia (decrease in heart rate by $25 \%$ from baseline).

For hemodynamic parameters, previous reports have been demonstrated that the observed hemodynamic alterations during UEGD procedure were transient and did not require any specific interventions. In this present study, the alteration of hemodynamic profiles including systolic and diastolic blood pressure, heart rate and oxygen saturation in both groups was similar to the previous studies. Although, mean oxygen saturation at the insertion of endoscope and mean heart rate at $10 \mathrm{~min}$ after the insertion of endoscope in sick patients were relatively lower than in nonsick patients, however, these alterations were mild, transient and easily managed without adverse sequelae. These hemodynamic alterations are likely a result of stress of the procedure. The significance of such stress with corresponding hemodynamic changes needs further investigations. Our previous study compared the anesthesia-related adverse event rate and the alteration of blood pressure and heart rate in UEGD procedure between elderly patients and younger patients. Anesthesia-related adverse event rate and alterations of blood pressure and heart rate during and immediately after UEGD procedure in the elderly patients are relatively high. However, these adverse events and the alterations of hemodynamic profiles in the elderly (aged $\geq 65$ years) patients did not higher than in the younger (aged $<65$ years) patients. All of these are mild and transient. ${ }^{21}$ The result of the study was comparable to the present study.

There are several limitations in this study. First, our study did not assess pre-procedure anxiety which has been shown to be a factor for successful completion of endoscopic procedures. ${ }^{14}$ Second, the endoscopic procedures were performed by variety of endoscopists including fellow in training. Therefore, the varied experience may have biased the result including the successful completion rate and the adverse event rate. Third, inaccurate and incomplete documentation of certain measures, as occurs with many chart reviews, also occurred in this study. Fourth, because the rate of serious adverse events in our series was none, further studies in larger prospective groups of patients are therefore needed. Overall, despite these limitations, we are confident, however, that these findings are generalizable to the practice of UEGD procedure in sick patients.

\section{Conclusion}

Topical pharyngeal anesthesia for UEGD procedure in sick patients was safe and effective with rare serious adverse events. Its efficacy in sick patients was not different or worse than in nonsick patients. Topical pharyngeal anesthesia might be a good technique for use in UEGD procedure even in the sick patients.

\section{References}

1. Lichtenstein DR, Jagannath S, Baron TH, et al. Sedation and anesthesia in GI endoscopy. Gastrointest Endosc. 2008;68(5):815-826.

2. Amornyotin S, Srikureja W, Chalayonnavin W, et al. Topical viscous lidocaine solution versus lidocaine spray for pharyngeal anesthesia in unsedated esophagogastroduodenoscopy. Endoscopy. 2009;41(7):581-586.

3. Axon ATR. Throat spray, sedation or anesthetic? Digestion. 2010;82(2):77-79.

4. Campo R, Brullet E, Montserrat A, et al. Topical pharyngeal anesthesia improves tolerance of upper gastrointestinal endoscopy: a randomized double blind study. Endoscopy. 1995;27(9):659-664.

5. Amornyotin S, Pranootnarabhal T, Chalayonnavin W, et al. Anesthesia for gastrointestinal endoscopy from 2005-2006 in Siriraj Hospital: a prospective study. Thai J Anesthesiol. 2007;33(2):93-101.

6. Amornyotin S, Prakanrattana U, Chalayonnavin W, et al. Anesthesia for gastrointestinal endoscopy in extremely elderly patients. Thai $J$ Anesthesiol. 2009;35(2):91-99.

7. Evans LT, Saberi S, Kim HM, et al. Pharyngeal anesthesia during sedated EGDs: is "the spray" beneficial? A meta-analysis and systematic review. Gastrointest Endosc. 2006;63(6):761-766.

8. Sorbi D, Chak A. Unsedated EGD. Gastrointest Endosc. 2003;58(1):102-110.

9. Amornyotin S, Chalayonnavin V, Kongphlay S. Recovery pattern and home-readiness after gastrointestinal endoscopy. J Med Assoc Thai. 2007;90(11):2352-2358.

10. Soma Y, Saito H, Kishibe T, et al. Evaluation of topical pharyngeal anesthesia for upper endoscopy including factors associated with patient tolerance. Gastrointest Endosc. 2001;53(1):14-18.

11. Mulcahy HE, Greaves RR, Ballinger A, et al. A double-blind randomized trial of low-dose versus high-dose topical anesthesia in unsedated upper gastrointestinal endoscopy. Aliment Pharmacol Ther. 1996;10(6):975-979.

12. Davis DE, Jones MP, Kubik CM. Topical pharyngeal anesthesia does not improve upper gastrointestinal endoscopy in conscious sedated patients. Am J Gastroenterol. 1999;94(7):1853-1856.

13. Dhir V, Swaroop VS, Vazifdar KF, et al. Topical pharyngeal anesthesia without intravenous sedation during upper gastrointestinal endoscopy. Indian J Gastroenterol. 1997;16(1):10-11.

14. Soma Y, Saito H, Kishibe T, et al. Evaluation of topical pharyngeal anesthesia for upper endoscopy including factors associated with patient tolerance. Gastrointest Endosc. 2001;53(1):14-18.

15. Wilkins T, Brewster A, Lammers J. Comparison of thin versus standard esophagogastroduodenoscopy. J Fam Pract. 2002;51(7):625-629.

16. Mulcahy HE, Kelly P, Banks MR, et al. Factors associated with tolerance to, and discomfort with, unsedated diagnostic gastroscopy. Scand J Gastroenterol. 2001;36(12):1352-1357.

17. McQuaid KR, Laine L. A systematic review and meta-analysis of randomized, controlled trials of moderate sedation for routine endoscopic procedures. Gastrointest Endosc. 2008;67(6):910-923.

18. Amornyotin S, Lertakayamanee N, Wongyingsinn $\mathrm{M}$, et al. The effectiveness of intravenous sedation in diagnostic upper gastrointestinal endoscopy. J Med Assoc Thai. 2007;90(2):301-306.

19. Sorbi D, Gostout CJ, Henry J, et al. Unsedated small-caliber esophagogastroduodenoscopy (EGD) versus conventional EGD: a comparative study. Gastroenterology. 1999;117(6):1301-1307.

20. Craig A, Hanlon J, Dent J, et al. A comparison of transnasal and transoral endoscopy with small-diameter endoscopes in unsedated patients. Gastrointest Endosc. 1999;49(3):292-296.

21. Amornyotin S, Kongphlay S. Anesthesia-related adverse event rate and alteration of blood pressure and heart rate during and immediately after unsedated esophagogastroduodenoscopy in elderly patients. $J$ Gastroenterol Hepatol Res. 2015;4(2):1474-1477. 